\title{
The Neurophysiology of Placebo Effect
}

\author{
Alessandro Piedimonte* \\ Department of Neuroscience, University of Turin, Italy
}

*Corresponding author: Alessandro Piedimonte, Ph.D. Department of Neuroscience, University of Turin, Turin, Italy, E-mail: ale.piedimonte@gmail.com

\section{Mini Review}

Volume 2 Issue 1

Received Date: February 27, 2018

Published Date: March 09, 2018

DOI: $10.23880 /$ cprj-16000108
Keywords: Placebo effect; Parkinson's disease

Abbreviations: PD: Parkinson's disease; CCK: Colecystokinin; rACC: Rostral Part of the Anterior Cingulate Cortex; DLPFC: Dorsolateral Prefrontal Cortex.

\section{Introduction}

Traditionally, the word placebo refers to an inert substance or treatment given to please the patient, in the absence of a real therapy. However this definition is not entirely correct. Indeed, recent advances in placebo research have conceptualized the placebo as the act of delivering a sham treatment in a therapeutic context full of physical and psychological elements [1].

Even if the placebo has no active properties itself, when a patient receives a placebo he/she can experience a true improvement in his ailment (e.g. reduction of pain perception or improvement in motor symptoms). This is the so called placebo effect.

Different studies have recently investigated the placebo effect in both clinical and experimental contexts identifying the psychological and neurobiological mechanisms underlying this phenomenon and increasing the knowledge of how to use the placebos in the clinical settings.

Different psychological models have been proposed to describe the placebo effect, namely classical conditioning and expectation. The first model explains these effects as the consequence of a learning process (as in the classical conditioning model). According to the classical conditioning model, after different pairings of a neutral stimulus (sound of a bell) with a sensory stimulus (e.g. food in the mouth) that produces itself a response (salivation), the presentation of the neutral stimulus alone produces the response. Following this model, the presentation of specific elements of the treatment (such as the shape of the pill) can produce conditioned analgesic responses if previously paired with active ingredient inside the pill.

According to the expectancy model, placebo and no placebo effects are triggered by cognitive expectations and beliefs held by the patient during a therapy. Different factors influence these expectations, such as verbal interactions with other patients and therapists, emotions during the treatment and previous experiences. For instance, the "stronger" the words accompanying a placebo cream supposed to relieve pain, the higher the expectation of pain relief, the stronger the placebo analgesia after the application of the placebo cream.

Besides this psychological approach, the focus of placebo research has been recently shifted toward the study of the neurobiological mechanisms, using pharmacological approaches and brain imaging techniques. The study of pain and Parkinson's disease (PD) has produced the best results.

Different pharmacological studies on pain have demonstrated that the administration of placebos activates the endogenous opioids and cannabinoids systems [2,3]. For example, after different exposures to opioid drugs (such as morphine), the administration of a placebo activate endogenous opioid that in turn produce relief of pain. This placebo analgesia can be reversed by using naloxone, an opioid antagonist. The involvement of the opioid system has also been investigated indirectly with two different drugs, proglumide and pentagastrin. Proglumide blocks colecystokinin (CCK), which has an anti-opioid action. Accordingly, proglumide potentiates opioid-mediated placebo analgesia. Conversely, pentagastrin facilitates CCK transmission, thus it blocks opioid-mediated placebo analgesia. In addition, proglumide has been found to antagonize nocebo hyperalgesia, thus suggesting that the nocebo effect is related to the activation of the CCK system. 
Another system involved in placebo analgesia is the cannabinoid system. In this case, after the administration of a non-opioid drug such as ketorolac, the administration of a placebo activates the CB1 cannabinoid receptors [4], with the consequent analgesia. This analgesic effect cannot be blocked by the administration of naloxone, but it can be antagonized by the CB1 antagonist rimonabant. Thus, if a patient was previously exposed to an opioid agent, placebo analgesia is mediated by opioid receptors, whereas the previous exposure to non-opioid drugs leads to a cannabinoid-mediated placebo analgesic response.

Neuroimaging studies have explored the brain regions involved in placebo analgesia and nocebo hyperalgesia [5]. Placebo analgesia has been found to be related to a reduced activation of brain areas involved in pain perception, the so-called "pain matrix", such as thalamus, insula, the rostral part of the anterior cingulate cortex (rACC), the dorsolateral prefrontal cortex (DLPFC), the primary somatosensory cortex, supramarginal gyrus, and left inferior parietal lobule. Interestingly, this reduced activation is similar to that following the administration of real analgesic drugs. Conversely, nocebo hyperalgesia seems to be related to an increased activity in the same areas of the pain matrix, such as the bilateral dorsal ACC, the left frontal and partietal operculum, the orbitofrontal cortex and the hippocampus.

Parkinson's disease (PD) has also provided important pieces of information on the physiological mechanisms of the placebo response [6]. Dopamine release has been found to be crucial in the placebo effect in PD patients. Dopaminergic activation takes place in the striatum, that is, in the same region which is involved in the pathophysiology of PD. In addition, by using singleneuron recording in awake PD patients, it was found that neuronal activity changes in the motor thalamus and in the sub thalamic nucleus after the administration of a placebo.

The study of placebo effects has not only improved our understanding of the complex relationship between cognitive processes and the underlying brain systems, but it has also provided important insights into the clinical setting $[7,8]$. For example, by exploiting the learning mechanisms of conditioning, it is possible to reduce the intake of drugs by administering drugs and placebo alternately. In addition, the recent findings highlight the important role of expectation, which can be enhanced during the doctor-patient relationship. Likewise, health professionals should strive to avoid nocebo effects by reducing negative expectation about possible adverse events of a therapy. Therefore, the study of placebo and nocebo effects seems to be of crucial importance both in modern neuroscience and in medical practice. In the former case, it represents an excellent model to study different brain mechanisms. In the latter, it represents a tool in the medical armamentarium to improve the outcome of any medical treatment.

\section{References}

1. Benedetti F (2014) Placebo effects. Oxford University Press 2nd (Edn.), pp: 416

2. Colloca L, Benedetti F (2005) Placebos and painkillers: is mind as real as matter? Nature Rev Neurosci 6(7): 545-552.

3. Bingel U, Wanigasekera $\mathrm{V}$, Wiech $\mathrm{K}, \mathrm{Ni}$ Mhuircheartaigh R, Lee MC, et al. (2011) The effect of treatment expectation on drug efficacy: imaging the analgesic benefit of the opioid remifentanil. Science Trans Med 3(70): 70ra14.

4. Benedetti F, Amanzio M, Rosato R, Blanchard C (2011) Non-opioid placebo analgesia is mediated by CB1 cannabinoid receptors. Nature Med 17(10): 1228-1230.

5. Wager TD, Rilling JK, Smith EE, Sokolik A, Casey KL, et al. (2004) Placebo induced changes in fMRI in the anticipation and experience of pain. Science 303(5661): 1162-1167.

6. de la Fuente-Fernandez R, Ruth TJ, Sossi V, Schulzer M, Calne DB, et al. (2001) Expectation and dopamine release: mechanism of the placebo effect in Parkinson's disease. Science 293(5532): 1164-1166.

7. Benedetti F (2010) The patient's brain: the neuroscience behind the doctor-patient relationship. Oxford University Press pp: 304.

8. Finniss DG, Kaptchuk TJ, Miller F, Benedetti F (2010) Biological, clinical, and ethical advances of placebo effects. Lancet 375(9715): 686-695. 